\title{
Efficiency of International Standard Serial Number Code as an Error Correcting Scheme
}

\author{
David Muriuki Gikunju ${ }^{1}$, Kamaku Peter Waweru ${ }^{1}$, Augustus Wali Nzomo² \\ ${ }^{1}$ Department of Pure and Applied Mathematics, Jomo Kenyatta University of Agriculture and Technology, Nairobi, Kenya \\ ${ }^{2}$ Department of Mathematics and Actuarial Science, South Eastern Kenya University, Kitui, Kenya
}

\section{Email address:}

gdavvie@gmail.com (D. M. Gikunju)

\section{To cite this article:}

David Muriuki Gikunju, Kamaku Peter Waweru, Augustus Wali Nzomo. Efficiency of International Standard Serial Number Code as an Error Correcting Scheme. Pure and Applied Mathematics Journal. Vol. 10, No. 1, 2021, pp. 1-8. doi: 10.11648/j.pamj.20211001.11

Received: July 30, 2020; Accepted: August 27, 2020; Published: February 10, 2021

\begin{abstract}
Error correcting coding is an effective technique of detecting and correcting errors which may occur due to environmental interference or physical defects such as human errors in the communication channels. The International Standard Serial Number code is internationally used for identifying the title of serial publications. This paper analyzes the efficiency of the international standard serial number code as an error correcting scheme. Moreover, the paper explores on the factors which affect the efficiency of any error correcting scheme. The study utilizes weight checksum technique to detect and correct error(s) in a code word. It is clear that ISSN code is not an efficient error coding scheme. ISSN code is only reliable in error detection. ISSN code can detect any error in the code iff the weight checksum equation does not hold. However, the code does not detect silent errors. The study develops a new efficient and robust modified ISSN code that is efficient in error detection and correction capabilities. The code has dual mechanism for error detection and correction in a code word. If the weight checksum equation does not hold and secondly, if the conditions for the generating equation do not hold. Modified ISSN code can detect and correct silent errors in a code word. Modified ISSN code is an efficient error coding scheme for it is efficient in error detection and correction capabilities.
\end{abstract}

Keywords: ISSN, Modified ISSN, Efficient, Error, Coding, Scheme

\section{Introduction}

Different communication channels have different error correcting coding schemes depending on the types of errors expected in a particular communication channel. An effective error coding requires an efficient scheme which is selected based on the characteristics of the specific communication channel.

\subsection{Factors Affecting the Efficiency of an Error Coding Scheme}

\subsubsection{Durability of the Entire Error Coding Scheme}

Durability of error coding scheme depends on the duration of time the entire coding system can stay before being exhausted. An efficient error coding scheme should last for a long period of time. The dictionary of the coding scheme should be large enough to be used for a longer period in its utilization.

\subsubsection{Precision of Code Words in an Error Coding Scheme}

The total number of digits in a code word in an efficient coding scheme should not be too large so as to lose its immediate utilization. A good $(n, q)$ error correcting code should have a considerable length $(n)$ for fast transmission of messages and large field $q$ to ensure wide transmission of a variety of information ( to ensure big dictionary). The code words of the coding scheme should have little similarities to each other. A good $(n, q)$ error correcting code should have a well define field $q$ to ensure precision in its usage. The length of all code words should be same.

\subsubsection{Reliability in Utilization of the Error Coding Scheme}

The primary principle of an error coding scheme is to assist the receiver of a message to get the true information intended 
by the sender, therefore, an efficient error coding scheme should guarantee high probability of accuracy of the messages received by the receiver. An error coding scheme can only guarantee a high probability of accuracy of the message if and only if it can be able to detect and correct error(s) which may occur. An efficient error coding scheme should, therefore, detect and correct all errors that may occur from the original message.

\subsection{Efficiency of the ISSN Code in Error Detection and Correction}

\subsubsection{Durability of the ISSN Code}

The International Standard Serial Number (ISSN) is a code that is internationally accepted for identifying the title of serial publications. From ISSN network statistics by January 2019, more than 2.5 million ISSNs had been issued. The number increases by approximately 60,000 to 70,000 ISSNs annually. However, about 130,000 ISSNs are changed and corrected annually. ISSN is only associated with the title of the publication. Therefore in case a publication is modified appreciably, a new ISSN has to be assigned. This means that if a publication title changes ten times the same publication has to be assigned ten new ISSN. It is clearly evident that the number of ISSNs changed and corrected is about double the number of ISSN increase per year.

Additionally, the entire ISSN coding scheme has a relatively small dictionary. The dictionary of 10,000, 000 code words and currently more than 2.5 million ISSNs has been issued by January 2019. This implies that with about 70,000 code words issued per year and 130,000 ISSNs changes and correction annually, the remaining $I S S N$ code words can last for maximum of 35 years. With high number of publications increase, currently ISSN coding scheme is not durable.

\subsubsection{Precision of the ISSN Code Words}

ISSN code is computed modulo 11, therefore the code is a finite field. ISSN code being a finite field of order 11, each element of the code word is supposed to be chosen from the set $F_{11}=\{0,1,2, \ldots, 9,10\}$ but the element 10 has two digits 1 and 0 , therefore, not applicable as an element of the code word. However, in the case when the check digit is 10 , an alphabetic letter is used. An alphabetic letter is used to ensure that the length of the ISSN codes remains eight. The usage of the alphabetic letter only in the check digit but not is an element of the entire code word makes the ISSN code to loss its brevity and precision. ISSN uses numeric digits except in check digit where alphabetic digit is used. Therefore, there is a lot of similarities of the elements from one code word to another.

\subsubsection{Reliability in the Utilization of the ISSN Code Words}

From the properties of ISSN code [12], it is clear that the following deductions holds;

1. ISSN code can detect any error in a code word if and only if the weight check sum equation does not hold.

2. ISSN code can detect and correct all single error in a code word if and if the position of the error is known.

3. ISSN can detect and correct all transposition errors in a code word even if the position of errors is unknown.

4. ISSN can detect and correct all double errors in a code word if and only if the position of errors in known.

5. ISSN cannot detect and correct silent error(s) in a code word.

6. ISSN code cannot correct jump transpose error, double error, jump twin error, phonetic error, twin error, omission, and insertion error in a code word.

Corollary 1.1. ISSN code is not an efficient error coding scheme.

Proof. It is clear that ISSN code is neither durable nor precise error correcting scheme. Moreover, it is clear that ISSN is not reliable in error correction for it only corrects a single error and a transposition error in a code word. Therefore, ISSN code is not efficient in error correction.

\section{Modified ISSN Code}

The Modified ISSN code is developed and generated by the use of functions, permutations and the properties of the existing ISSN code. The code is developed on the basis of a good communication channel and an efficient error coding scheme.

\subsection{Development and Generation of Modified ISSN}

1. The Modified ISSN code is an alphanumerical code, that is, it is made of alphabetical digits and numerical digits.

2. The Modified ISSN code is a finite field of order 31 where each digit is chosen from the set $F_{31}$. The set $F_{31}$ is the set $\mathbb{Z}_{31}=\{0,1,2, \ldots, 30\}$. Each code word of the modified ISSN code has a length of 8 digits, where each digit chosen from the set $F_{31}$. Alphabetical digit $O$ and $I$ are omitted, for $O$ resembles numerical digit 0 and $I$ resembles 1 hence to avoid confusion. Thus the set $F_{31}=\{0,1,2, \ldots, 8,9, A, B, C, \ldots, U, V, W\}$ without alphabetical digits $I$ and $O$. The main reason of using alphabetical digits is to replace two digits numerical digits hence avoiding confusing and enhancing preciseness.

3. The elements in each code word is generated by the generating equation defined by $x_{i+1}=3 x_{i}+$ $5(\bmod 31), i=1,2, \ldots, 6$ except the last digit of the code word. The last digit of code word is the check digit hence is computed. The first term(digit) of the generating equation is chosen randomly from the $F_{31} \cong$ $\mathbb{Z}_{31}$.

4. It is not a must for the generating equation defined by $x_{i+1}=3 x_{i}+5(\bmod 31), i=1,2, \ldots, 6$ to start at the beginning of a code word. The sequence generated by of the generating equation can also be terminated in a code word. In the case where the sequence is terminated, digit 0 is inserted after the last digit of the terminated sequence and before the first digit of the other generating 
equation . The first term(digit) of the other generating equation is also chosen randomly from the $F_{31} \cong \mathbb{Z}_{31}$.

5. The digit 0 does not have to necessarily be used when a sequence generated by the generating equation has been terminated, it may be used as an element of the code word itself. That is, digit 0 may be generated by the generating equation or may be at the beginning of the generating equation. In this case, digit 0 acts as a neutral digit. This implies that the generated sequence does not necessarily terminate once there is a digit 0 .

6. In the case where the generating equation does not start at the beginning of a code word, the first digit of sequence is repeated from the beginning of a code word until where the start digit of the generating equation starts. Moreover, it is not a must for the generating equation to start at the beginning immediately after digit 0 in case the generated sequence had been terminated.
In the case where the generating equation does not start after digit 0 , the first digit of the new sequence is repeated after digit 0 until where the start digit of generating equation starts.

7. ISSN codes are only associated with the title of a publication, in case changes and corrections are made to the title of a publication, the code word will have an extra digit called a blind digit. A blind digit is separated from the check digit by a hyphen. A blind digit does not affect the weight check sum of the entire code word. It is given depending on the number of changes made in the title of a publication. Importance of the blind digit is to help to know the number of times the changes and corrections have been made to a title of a publication.

8. The eight digits of the modified ISSN code must satisfy $\sum_{i=1}^{8} j x_{i} \equiv 0(\bmod 31)$.

Examples of modified ISSN code;

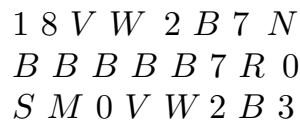

$\begin{array}{lllllll}11 & 1 & 1 & 8 & W & C\end{array}$

$3 E G N 918 L-2$

$\checkmark W 2 B 0 T Q D$
$L 30 R J U 0 U$

Where

Table 1. Elements of modified ISSN code.

\begin{tabular}{llllllllll}
\hline $\mathbf{0}$ & $\mathbf{1}$ & $\mathbf{2}$ & $\mathbf{3}$ & $\mathbf{4}$ & $\mathbf{5}$ & $\mathbf{6}$ & $\mathbf{7}$ & $\mathbf{8}$ & $\mathbf{9}$ \\
$\mathrm{A}=10$ & $\mathrm{~B}=11$ & $\mathrm{C}=12$ & $\mathrm{D}=13$ & $\mathrm{E}=14$ & $\mathrm{~F}=15$ & $\mathrm{G}=16$ & $\mathrm{H}=17$ & $\mathrm{~J}=18$ & $\mathrm{~K}=19$ \\
$\mathrm{~L}=20$ & $\mathrm{M}=21$ & $\mathrm{~N}=22$ & $\mathrm{P}=23$ & $\mathrm{Q}=24$ & $\mathrm{R}=25$ & $\mathrm{~S}=26$ & $\mathrm{~T}=27$ & $\mathrm{U}=28$ & $\mathrm{~V}=29$ \\
\hline
\end{tabular}

\subsection{Number of Code Words in Modified ISSN Code}

Proposition 2.1. Modified ISSN code has a dictionary of upper bound of 28, 644, 217.

Proof. The Modified ISSN code is finite field of order 31 where each digit is chosen from the set $F_{31} \cong \mathbb{Z}_{31}$. Each code word of the modified ISSN code has a length of 8 symbols,where each symbol is chosen from the set $F_{31}$. Moreover, the eighth digit of the modified ISSN code is the check digit which is computed on the basis of weight checksum equation. The elements in each code word is generated by the generating equation defined by $x_{i+1}=$ $3 x_{i}+5(\bmod 31) i=1,2,3, \ldots, 6$. It is not a must for the generating formula for the generation of the elements of a code word to start at the begin of the code word. Total numbers of the code words depends on the generating equation and digit 0 for the termination of the generated sequence. Digit 0 for the termination of the generating equation can occur from the second digit of a code word to the seventh digit of a code word. There are 31 ways of choosing the first digit of a code word, two ways of choosing the second digit, that is, through generating equation or repetition if generating equation does not start in the beginning of a code word. From the third digit to the seventh digit there are three ways of choosing each digit. There is only one way of choosing the eighth digit for it is computed to satisfy the weight checksum equation. Moreover, if there is a digit 0 in second digit, there are 31 ways of choosing the third digit, similar to the fourth digit until the seventh digit. Therefore there are $31 \times 2 \times 3^{5}$ or $31^{5}$ ways of choosing a code word, hence the dictionary of the modified ISSN code is $15066+28,629,151=28,644,217$.

\subsection{Calculation of the Check Digit in a Modified ISSN Code Word}

Let the code word for modified ISSN be $\mathrm{X}=\mathrm{x}_{1}, \mathrm{x}_{2}, \ldots, \mathrm{x}_{7}$ without the check digit. To compute the check digit, $\mathrm{x}_{8}$, since the code word digits $\mathrm{x}_{1}, \mathrm{x}_{2}, \ldots, \mathrm{x}_{7}$ are known, calculate $\sum_{i=1}^{7} j x_{i=9-i}$. Let $\xi=\sum_{i=1}^{7} j x_{i=9-i}$ then $\mathrm{x}_{8}+\xi \equiv 0(\bmod 31)$. Now since 0 is additive identity of $\mathbb{Z}_{\mathrm{n}}$ , therefore $\mathrm{x}_{8}$ is the additive inverse of $\xi(\bmod 31)$. Thus $\mathrm{x}_{8} \equiv-\xi(\bmod 31)$.

Example 2.1. Calculate $x_{8}$ for the ISSN code word $18 V W 2 B 7 x_{8}$. To compute $x_{8}$, calculate $\xi=$ 
$\sum_{i=1}^{7} j x_{i}=443(\bmod 31)=9(\bmod 31)$. Hence $x_{8}=$ $9^{-1}(\bmod 31)=22=N$.

\section{Error Detection in Modified ISSN Code}

Modified ISSN code is an improved error coding scheme in term of error detection and correction capabilities. Let $X=x_{1}, x_{2}, \ldots, x_{8}$ be the code word for modified ISSN, then the weight checksum is computed $\sum_{i=1}^{8} j x_{i=9-i} \equiv \bmod 31$. If $\sum_{i=1}^{8} j x_{i} \neq 0(\bmod 31)$ then the code has detected error(s). Moreover, Let the code word for modified ISSN be $X=x_{1}, x_{2}, \ldots, x_{8}$, then $x_{2}=\left(3 x_{1}+5\right)(\bmod 31), x_{3}=$ $\left(3 x_{2}+5\right)(\bmod 31), \quad, \ldots x_{7}=\left(3 x_{6}+5\right)(\bmod 31)$. If $x_{i} \neq$ $\left(3 x_{i-1}+5\right)(\bmod 31), i=2,3, . ., 7$ then the code has detected error(s). An ISSN code word has a length of only eight digits unless there is change of the title of the publication where the code word has nine digits, therefore, if the code has a length of more or less than eight then there is an error.

Remark 3.1. if $x_{i} \neq\left(3 x_{i-1}+5\right)(\bmod 31), i=2,3, . ., 7$ does not necessarily indicate an error in the code word for there are two occasions where $x_{i} \neq\left(3 x_{i-1}+5\right)(\bmod 31)$, $i=2,3, . ., 7$ will occur but no error has occurred in the code word:

a. In case there is a digit 0 indicating the termination of the generating equation $x_{i+1}=3 x_{i}+5(\bmod 31), i=1,2, \ldots, 6$

b. In case there is repetition of a digit indicating that the generating equation $x_{i+1}=3 x_{i}+5(\bmod 31), i=1,2, \ldots, 6$ has not begun in the beginning of the code word or after digit 0 when there is termination of the generating equation. The code word has to satisfy the checksum weight equation $\sum_{i=1}^{8} j x_{i} \equiv 0(\bmod 31)$.

\subsection{Single Error Detection in a Modified ISSN Code Word}

Proposition 3.1. The Modified ISSN code detects any single error in the Modified ISSN code word.

Proof. Suppose $X=x_{1}, x_{2}, \ldots, x_{8}$ is the modified ISSN code word and $Y=x_{1}, x_{2}, \ldots, x_{\tau-1}, y_{\tau}, x_{\tau+1}, \ldots, x_{8}$ with $y_{\tau}=x_{\tau}+\alpha \quad \alpha \neq 0$ is the modified ISSN code word with a single error that has occurred in digit $x_{\tau}, 1 \leq \tau \geq 8$. Then

$$
\begin{aligned}
& \left(\sum_{i=1}^{8} j y_{i}\right) \\
& \left(\sum_{i=1}^{8} j x_{i}\right)+(9-\tau) \alpha \neq 0(\bmod 31) \\
& (9-\tau) \alpha \neq 0(\bmod 31)
\end{aligned}
$$

Moreover, $y_{\tau} \neq\left(3 x_{\tau-1}+5\right)(\bmod 31)$. Therefore the single error is detected.

Proposition 3.2. The Modified ISSN code detects silent error in a code word.

Proof. Suppose a single error has occurred in a modified ISSN code word but $\sum_{i=1}^{8} j x_{i} \equiv 0(\bmod 31)$. Then if $Y=x_{1}, x_{2}, \ldots, x_{\tau-1}, y_{\tau}, x_{\tau+1}, \ldots, x_{8}$ with $y_{\tau}=x_{\tau}+\alpha \quad \alpha \neq$ 0 is the modified ISSN code word with a single error that has occurred in the position $x_{\tau}$ then

$$
\begin{aligned}
& \left(\sum_{i=1}^{8} j y_{i}\right) \\
& \left(\sum_{i=1}^{8} j y_{i}\right)+(9-\tau) \alpha \equiv 0(\bmod 31) \\
& (9-\tau) \alpha \equiv 0(\bmod 31)
\end{aligned}
$$

This implies that either $9-\tau$ or $\alpha$ is a multiple of 31 or 0 . Since $9-\tau$ cannot be a multiple of 31 or 0 for $1 \leq \tau \leq 8$ hence $\alpha=0$ and no error. However, there is a single error in the code word then there exist a digit of the code word $x_{i} \neq$ $\left(3 x_{i-1}+5\right)(\bmod 31)$. Therefore $x_{\tau} \neq\left(3 x_{\tau-1}+5\right)(\bmod 31)$ and hence the silent error detected. Conversely, Suppose a single error has occurred in modified ISSN code but $x_{i}=$ $\left(3 x_{i-1}+5\right)(\bmod 31)$ for $i=2,3, . ., 7$ then $\sum_{i=1}^{8} j x_{i=9-i} \neq$ $0(\bmod 31)$ then the silent error is detected.

Proposition 3.3. The Modified ISSN code corrects the single errors in a code word.

Proof. Suppose $X=x_{1}, x_{2}, \ldots, x_{8}$ is the modified ISSN code word and $Y=x_{1}, x_{2}, \ldots, x_{\tau-1}, y_{\tau}, x_{\tau+1}, \ldots, x_{8}$ with $y_{\tau}=x_{\tau}+\alpha \quad \alpha \neq 0$ is the modified ISSN code word with a single error that has occurred in digit $y_{\tau}, 1 \leq \tau \geq 8$. The most important thing is the code to detect the position of the digit with error. From second digit to the second last digit of the code word is generated by the generating equation $x_{\tau+1}=3 x_{\tau}+5(\bmod 31)$ unless there is a repetition of digits or digit 0 indicating termination of the generating equation. By use of the generating equation $x_{\tau+1}=3 x_{\tau}+5(\bmod 31)$ the digit $y_{\tau}=x_{\tau}+\alpha \quad \alpha \neq 0$ is detected by $y_{\tau} \neq\left(3 x_{\tau-1}+\right.$ $5)(\bmod 31)$. After the position of $y_{\tau}$ is detected, the error is corrected by the computing $y_{\tau}=\left(3 x_{\tau-1}+5\right)(\bmod 31)$ and $y_{\tau}=\left(\frac{x_{\tau+1}-5}{3}\right)(\bmod 31)$. In case $x_{\tau+1}<5$ or $\left(x_{\tau+1}-5\right)$ is not divisible by 3 then

$$
\begin{gathered}
3 y_{\tau} \equiv x_{\tau+1}-5(\bmod 31) \\
3^{-1} y_{\tau}=3^{-1} \times\left(\left(x_{\tau+1}-5\right)(\bmod 31)\right) \\
y_{\tau}=21 \times\left(\left(x_{\tau+1}-5\right)(\bmod 31)\right) \\
\text { when } x_{\tau+1}<5 \\
y_{\tau}=21 \times\left(\left(31+\left(x_{\tau+1}-5\right)\right)(\bmod 31)\right)
\end{gathered}
$$

Then the weight checksum equation $\sum_{i=1}^{8} j x_{i=9-i} \equiv$ 
$0(\bmod 31)$ is tested whether it holds. Additionally, when the error has been detected and the position of the error is known. Then the error can be corrected by

$$
\begin{gathered}
\sum_{i=1}^{8} j x_{i \neq \tau}+(9-\tau) y_{\tau} \equiv 0(\bmod 31) \\
(9-\tau) y_{\tau} \equiv-\left[\left(\sum_{i=1}^{8} j x_{i}\right)(\bmod 31)\right] \\
y_{\tau}=(9-\tau)^{-1} \times-\left[\left(\sum_{i=1}^{8} j x_{i \neq \tau}\right)(\bmod 31)\right]
\end{gathered}
$$

Since $y_{\tau}=x_{\tau}+\alpha, \alpha=0$ hence $y_{\tau}=x_{\tau}$ yielding the original modified ISSN code word $X=x_{1}, x_{2}, \ldots, x_{8}$.

Example 3.1. Let $18 \mathrm{VW} 2 \mathrm{~B} 7 \mathrm{~N}$ be the modified ISSN code word and $18 \mathrm{VWCB} 7 \mathrm{~N}$ be the modified ISSN code word with a single error at $x_{5}$. By

$$
\begin{aligned}
& \sum_{i=1}^{8} j x_{i} \neq 0-i(\bmod 31) \\
& 505 \neq 0(\bmod 31) \\
& 9 \neq 0(\bmod 31)
\end{aligned}
$$

therefore the single error is detected. By use of the generating equation $x_{i+1}=\left(3 x_{i}+5\right)(\bmod 31)$ the position of the error is found. The error is at $x_{5}$ for

$$
\begin{aligned}
& C=12 \neq(3 W+5)(\bmod 31) \\
& 12 \neq 95(\bmod 31)
\end{aligned}
$$

the error is corrected by the computing $y_{\tau}=\left(3 x_{\tau-1}+\right.$ $5)(\bmod 31)$ and $y_{\tau}=\left(\frac{x_{\tau+1}-5}{3}\right)(\bmod 31)$

$$
\begin{aligned}
& x_{5}=\left(3 x_{4}+5\right) \Rightarrow x_{5}=(3 V+5)(\bmod 31) \\
& x_{5}=(90+5)(\bmod 31) \\
& x_{5}=2 \text { and } \\
& x_{5}=\left(\frac{B-5}{3}\right)(\bmod 31) \\
& x_{5}=\left(\frac{11-5}{3}\right)(\bmod 31) \\
& x_{5}=2
\end{aligned}
$$

Hence the error is corrected.

Remark 3.2. The Modified ISSN is more efficient in single error detection and correction than ISSN code.

\subsection{Transposition Error Detection and Correction in a Modified ISSN Code Word}

Proposition 3.4. The Modified ISSN code detects all transposition error in a code word.

Proof. Suppose $X=x_{i}, i=1,2, \ldots, 8$ is the modified ISSN code word and $Y=x_{1}, x_{2}, \ldots, x_{\beta}, x_{\tau}, \ldots, x_{8}$ with $x_{\tau}$ and $x_{\beta}$ be exchanged digits of the modified ISSN code word. Then

$$
\begin{aligned}
& \left(\sum_{i=1}^{8} j y_{i}\right) \\
& \left(\sum_{i=1}^{8} \sum_{i \neq \tau, \beta} j x_{i}\right)+(\tau-\beta) x_{\tau}+(\beta-\tau) x_{\tau} \neq 0(\bmod 31) \\
& (\tau-\beta)\left(x_{\tau}-x_{\beta}\right) \neq 0(\bmod 31)
\end{aligned}
$$

provided $\tau \neq \beta$ and $x_{\tau} \neq x_{\beta}$. Therefore, transposition error of digits $x_{\tau}$ and $x_{\beta}$ is detected. Alternatively, Suppose $X=x_{i}, i=1,2, \ldots, 8$ is the modified ISSN code word and $Y=x_{1}, x_{2}, \ldots, x_{\beta}, x_{\tau}, \ldots, x_{8}$ with $x_{\tau}$ and $x_{\beta}$ be exchanged digits of the ISSN code word. Then

$$
\begin{aligned}
& x_{\beta} \neq\left(3 x_{\tau}+5\right)(\bmod 31) \text { and } \\
& x_{\tau} \neq\left(\frac{x_{\beta}-5}{3}\right)(\bmod 31)
\end{aligned}
$$

but

$$
\begin{aligned}
& x_{\tau}=\left(3 x_{\beta}+5\right)(\bmod 31) \text { and } \\
& x_{\beta}=\left(\frac{x_{\tau}-5}{3}\right)(\bmod 31)
\end{aligned}
$$

Therefore transposition error of digits $x_{\tau}$ and $x_{\beta}$ detected.

Corollary 3.1. The Modified ISSN code corrects all transposition errors in the code word.

Proof. From the above prove (detection of transpose error) it is clear that

$$
\begin{aligned}
& x_{i}=\left(3 x_{i-1}+5\right)(\bmod 31) i=2,3, \ldots, 7 \\
& x_{\beta} \neq\left(3 x_{\tau}+5\right)(\bmod 31) \\
& x_{\tau} \neq\left(\frac{x_{\beta}-5}{3}\right)(\bmod 31)
\end{aligned}
$$

but

$$
\begin{aligned}
& x_{\tau}=\left(3 x_{\beta}+5\right)(\bmod 31) \\
& x_{\beta}=\left(\frac{x_{\tau}-5}{3}\right)(\bmod 31)
\end{aligned}
$$

Thus implying there is a transpose error which can be corrected by exchanging the digits $x_{\tau}$ and $x_{\beta}$.

Example 3.2. Let1 $8 V W 2 B 7 N$ be the modified ISSN code word and $18 \mathrm{VWB} 27 \mathrm{~N}$ be the modified ISSN code word with adjacent transposed error at $x_{5}$ and $x_{6}$. Consider the new modified ISSN code word $18 \mathrm{VWB} 27 \mathrm{~N}$, the weight checksum equation $\left.\sum_{i=1}^{8} j x_{i} \neq 0(\bmod 31)\right) \cdot \tau=$ $5, \beta=6, x_{\tau}=11, x_{\beta}=2(\tau-\beta)\left(x_{\tau}-x_{\beta}\right) \neq 0(\bmod 31) \Rightarrow$ $(5-6)(11-2)=-9 \neq 0(\bmod 31)$. Moreover, $2 \neq(3 \times 11+$ $5)(\bmod 31)$ for $(3 \times 11+5)(\bmod 31)=38(\bmod 31)=7$ and $11 \neq\left(\frac{2-5}{3}\right)(\bmod 31)$ but $11=(2 \times 3+5)(\bmod 31)$ and $2=\left(\frac{11-5}{3}\right)$. Hence the transposed error detected. Since the transposed error is detected the code can be corrected by transposing the digits yielding $18 \mathrm{VW} 2 \mathrm{~B} 7 \mathrm{~N}$ by which the weight checksum equation holds. Hence the error 
corrected.

Remark 3.3. The Modified ISSN is more efficient in transpose error detection and correction than ISSN code.

\subsection{Jump Transposition Error Detection and Correction in Modified ISSN Code}

Proposition 3.5. The Modified ISSN code detects all jump transposition error in a code word.

Proof. Suppose that $X=x_{1}, x_{2}, x_{3}, x_{4}, x_{5}, x_{6}, x_{7}, x_{8}$ is a modified ISSN code word and $Y=$ $x_{1}, x_{2}, x_{5}, x_{4}, x_{3}, x_{6}, x_{7}, x_{8}$ be modified ISSN code word with jump transposition error. The check sum of $X$ and $Y$ are $8 x_{1}+7 x_{2}+6 x_{3}+5 x_{4}+4 x_{5}+3 x_{6}+2 x_{7}+x_{8}$ and $8 x_{1}+7 x_{2}+6 x_{5}+5 x_{4}+4 x_{3}+3 x_{6}+2 x_{7}+x_{8}$ respectively. Assume that $X$ and $Y$ are multiple of 31 that is both are $\sum_{i=1}^{8} j x_{i} \equiv 0(\bmod 31)$. Consider $X-Y$. After cancellation

$$
\begin{aligned}
X-Y & =6 x_{3}-6 x_{5}+4 x_{5}-4 x_{3} \\
& =2 x_{3}-2 x_{5}=2\left(x_{3}-x_{5}\right) .
\end{aligned}
$$

Since $x_{3}-x_{5}$ is $-30 \leq x_{3}-x_{5} \leq 30$. The only multiple of 31 between -30 and 30 is zero. Therefore $x_{3}-x_{5}=0$, then $x_{3}=x_{5}$ meaning no error has occurred - This is a contradiction. Alternatively, Suppose that $X=$ $x_{1}, x_{2}, x_{3}, x_{4}, x_{5}, x_{6}, x_{7}, x_{8}$ is a modified ISSN code word and $Y=x_{1}, x_{2}, x_{5}, x_{4}, x_{3}, x_{6}, x_{7}, x_{8}$ be the modified ISSN code word with jump transposition error. Consider $Y=$ $x_{1}, x_{2}, x_{5}, x_{4}, x_{3}, x_{6}, x_{7}, x_{8}$ then the error can be detected by $x_{4} \neq\left(3 x_{5}+5\right)(\bmod 31)$, and $x_{4} \neq\left(\frac{x_{3}-5}{3}\right) \bmod 31$ but $x_{4}=\left(3 x_{3}+5\right)(\bmod 31)$ and $x_{4}=\left(\frac{x_{5}-5}{3}\right)(\bmod 31)$. Hence jump transposition error detected.

Corollary 3.2. The Modified ISSN code corrects all jump transposition error in a code word.

Proof. It is clear that once the jump transposition error has been detected can be corrected. Suppose that $X=$ $x_{1}, x_{2}, x_{3}, x_{4}, x_{5}, x_{6}, x_{7}, x_{8}$ is a modified ISSN code word and $Y=x_{1}, x_{2}, x_{5}, x_{4}, x_{3}, x_{6}, x_{7}, x_{8}$ be the modified ISSN code word with jump transposition error. Consider $Y=$ $x_{1}, x_{2}, x_{5}, x_{4}, x_{3}, x_{6}, x_{7}, x_{8}$ once the error is detected by $x_{4} \neq$ $\left(3 x_{5}+5\right)(\bmod 31), x_{4} \neq\left(\frac{x_{3}-5}{3}\right)(\bmod 31)$ but $x_{4}=\left(3 x_{3}+\right.$ $5)(\bmod 31)$ and $x_{4}=\left(\frac{x_{5}-5}{3}\right)(\bmod 31)$. This implies that the error can be corrected by transposing $x_{3}$ and $x_{5}$ and then test whether the weight checksum equation holds and the generating equation $x_{i}=\left(3 x_{i-1}+5\right)(\bmod 31) i=2,3, \ldots, 7$ holds.

Example 3.3. Let $18 \mathrm{VW} 2 \mathrm{~B} 7 \mathrm{~N}$ be the modified ISSN and $182 W V 27 \mathrm{~N}$ be the modified ISSN code word with jump transposed error at $x_{3}$ and $x_{5}$.The error is detected by the fact that $x_{4} \neq\left(3 x_{5}+5\right)(\bmod 31)$, and $x_{4} \neq$ $\left(\frac{x_{3}-5}{3}\right)(\bmod 31) 1$ but $x_{4}=\left(3 x_{3}+5\right)(\bmod 31)$ and $x_{4}=\left(\frac{x_{5}-5}{3}\right)(\bmod 31)$ therefore the jump transposition error can be corrected by exchanging $x_{3}$ and $x_{5}$ yielding $18 \mathrm{VW} 2 \mathrm{~B} 7 \mathrm{~N}$ in which both $x_{i}=\left(3 x_{i-1}+\right.$
5) $(\bmod 31) i=2,3, \ldots, 7$ and $\sum_{i=1}^{8} j x_{i=9-i} \equiv 0(\bmod 31)$ hold.

\subsection{Double Error Detection and Correction in Modified ISSN Code}

Proposition 3.6. The Modified ISSN code detects and corrects all double error in the code word.

Proof. Suppose $X=x_{i}, i=1,2, \ldots, 8$ is the modified ISSN code word and $Y=x_{1}, x_{2}, \ldots, x_{\tau-1}, x_{\tau}, x_{\beta}, x_{\beta+1} \ldots, x_{8}$ be the modified ISSN code word with double error in digit $x_{\tau}$ and $x_{\beta}$. Without loss of generality $Y=\sum_{i=1}^{8} j x_{i=9-i} \neq$ $0(\bmod 31)$ and error detected in the code word $Y$. Suppose there is no error in the $Y$ then $Y=\sum_{i=1}^{8} j x_{i=9-i} \equiv$ $0(\bmod 31)$ and $x_{i}=\left(3 x_{i-1}+5\right) \bmod 31 i=2,3, \ldots, 7$ but this not the case. Since there is an error in $Y$, the double error is detected by

$$
\begin{aligned}
& x_{\beta} \neq\left(3 x_{\tau}+5\right)(\bmod 31) \\
& x_{\tau} \neq\left(\frac{x_{\beta}-5}{3}\right)(\bmod 31)
\end{aligned}
$$

moreover,

$$
\begin{aligned}
& x_{\tau} \neq\left(3 x_{\tau-1}+5\right)(\bmod 31) \\
& x_{\beta} \neq\left(\frac{x_{\beta+1}-5}{3}\right)(\bmod 31)
\end{aligned}
$$

but the rest $x_{i}=\left(3 x_{i-1}+5\right)(\bmod 31)$ holds. Since $x_{\tau-1}=$ $\left(3 x_{\tau-2}+5\right)(\bmod 31)$ and $x_{\beta+1}=\left(\frac{x_{\beta+2}-5}{3}\right)(\bmod 31)$, the twin error in $Y$ is corrected by making $x_{\tau}=\left(3 x_{\tau-1}+\right.$ $5)(\bmod 31)$ and $x_{\beta}=\left(\frac{x_{\beta+1}-5}{3}\right)(\bmod 31)$ then test whether the weight checksum equation holds.

Example 3.4. Let $18 \mathrm{VW} 2 \mathrm{~B} 7 \mathrm{~N}$ be the modified ISSN code word and $18 \mathrm{~V} 5 \mathrm{CB} 7 \mathrm{~N}$ be the modified ISSN code word with double error at $x_{3}$ and $x_{4}$. Since the weight checksum equation does not hold, the error is detected in the code word. The double error is detected by

$$
\begin{array}{ll}
C \neq(3 \times 5+5)(\bmod 31) & C=12 \neq 20(\bmod 31) \\
5 \neq\left(\frac{C-5}{3}\right)(\bmod 31) & 5 \neq\left(\frac{12-5}{3}\right)(\bmod 31) \\
5 \neq(3 \times V+5)(\bmod 31) & 5 \neq 30(\bmod 31)
\end{array}
$$

and

$$
\begin{aligned}
C & \neq\left(\frac{B-5}{3}\right)(\bmod 31) \\
12 & \neq\left(\frac{11-5}{3}\right)(\bmod 31)
\end{aligned}
$$

Since

$$
\begin{gathered}
V=(3 \times 8+5)(\bmod 31) \\
29=(3 \times 8+5)(\bmod 31)
\end{gathered}
$$


and

$$
\begin{aligned}
B & =\left(\frac{7-5}{3}\right)(\bmod 31) \\
11 & =3^{-1} \times 2(\bmod 31) \\
11 & =21 \times 2(\bmod 31) \\
11 & =42(\bmod 31)
\end{aligned}
$$

then

$$
\begin{aligned}
& x_{\tau}=(3 \times V+5)(\bmod 31) \\
& x_{\tau}=(3 \times 29+5)(\bmod 31) \\
& x_{\tau}=30=W
\end{aligned}
$$

and

$$
\begin{aligned}
& x_{\beta}=\left(\frac{B-5}{3}\right)(\bmod 31) \\
& x_{\beta}=\left(\frac{11-5}{3}\right)(\bmod 31) \\
& x_{\beta}=2
\end{aligned}
$$

Therefore the code has detected and corrected double errors in the code word.

\section{Efficiency of the Modified ISSN in Error Detection and Correction}

Proposition 4.1. Modified ISSN code is an efficient error coding scheme.

Proof. Without loss of generality, modified ISSN code is efficient in error detection and correction. Moreover, modified ISSN has a blind digit which shows the number of corrections and modification done of the title of publication. ISSN coding scheme is only associated with the title of the publication. Therefore in case a publication is modified appreciably, only the blind digit changes. This means that if a publication is modified ten times the modified ISSN code shows that the title of the publication has being changed ten times. Moreover, modified ISSN code is a finite field where its elements are precisely selected and therefore an efficient coding scheme.

\section{Conclusion}

The study has shown that ISSN code is not an efficient error coding scheme. ISSN code is only efficient in error detection for it only reliable in error detection. ISSN code can detect any error in the code iff the weight checksum equation does not hold. However, the code does not detect silent errors. Modified ISSN code is efficient in error detection and correction capabilities. The code has dual mechanism of detection of errors in a code word. First, if the weight checksum equation does hold and secondly, if the generating equation does not hold. Modified ISSN code can detect and correct silent error in a code word. Modified ISSN code is an efficient error coding scheme for it is efficient in error detection and correction capabilities. Moreover, the code has a relatively big dictionar.

\section{References}

[1] Achalare, R. A., Patil, S. V., \& Patil, S. S. (2014). Significance of ISSN and ISBN in Publications. Current Pharma Research, 5 (1).

[2] Brown, S. (2003). Assignment of International Standard Serial Numbers (ISSNs) to Online Resources: a research project. Serials, 16 (3).

[3] Doumen, J. M. (2003). Some Applications of Coding Theory in Cryptography. Eindhoven University Press, Germany, Germany.

[4] Egghe, L. (2005). The Coding of the ISBN. Retrieved from: http://en.scientificcommons.org /leo_ egghe, Date accessed: 6th May 2010.

[5] Egghe, L. and Ronald, R. (2005). On the detection of double errors in isbn and issn-like codes.Retrieved from: http://en.scientific commons. org/leo_egghe,Dateaccessed: 6th May 2010.

[6] Eric, W. (2010). ISBN code. Retrieved from: http://mathworld.wolfram.com/ISBN.html, Date accessed: 5th May 2010.

[7] Huffman, W. C., \& Pless, V. (2010). Fundamentals of Error-Correcting Codes. Cambridge university press.

[8] ISSN International Centre (2003). ISSN manual: International Standard Serial Number. Paris: ISSN International Centre.

[9] ISSN International Centre (2016). ISSN International Centre activity report for 2016. Paris: ISSN International Centre.

[10] Kamaku,W. (2012). Error Detection and Correction on the International Standard Book Number. American International Journal of Contemporary Research, 2 (2): 150152 .

[11] Lowery, J. (2014, June 26). International Standard Serial Number (ISSN). Retrieved from http://www.bl.uk/issn\#.

[12] Muriuki, D. G., Waweru, P. K., \& Wali, A. N. (2019). Properties of the International Standard Serial Number. Journal of Basic and Applied .Vol. 47, No. 1 pp 117-129.

[13] National Information Standards Organization (U.S.), \& American National Standards Institute. (1995). International standard serial numbering (ISSN). Bethesda, Md., U.S.A: NISO Press.

[14] Nyaga, L. and Cecilia, M. (2008). Increasing Error Detection and Correction Efficiency in the ISBN. Discovery and Innovation, 20: 3-4. 
[15] Oury, C. (2016). Revising the ISSN: involving stakeholders to adapt a bibliographic standard to its everchanging environment.

[16] Singh, J., \& Singh, J. (2012, January). A comparative study of error detection and correction coding techniques. In 2012 Second International Conference on Advanced Computing \& Communication Technologies (pp. 187189). IEEE.

[17] Sivan, S., Katta, S., Venkatesulu, P., \& Nayaka, R. J. (2013, April). FPGA implementation of low bandwidth ECC code. In 2013 IEEE Conference on Information \& Communication Technologies (pp. 468-472). IEEE.
[18] Skrzypczak, J. (2014). International Standard Serial Number (ISSN).

[19] Stein, W. (2009). Elementary Number theory: Primes, Congruences, and Secrets. Undergraduate Texts in Mathematics.

[20] Todd, K. M. (2005). Error Correction Coding: Mathematical Methods and Algorithms John Wiley \& Sons Inc.

[21] Wachira, L. (2015). Error Detection and Correction on the Credit Card Number Using Luhn Algorith. Master's thesis, J.K.U.A.T, Juja, Kenya. 2 (2): 150152. 